\title{
LÓGICA DE LA GESTIÓN AMBIENTALMENTE ADECUADA Y PATRÓN DE DESARROLLO SUSTENTABLE EN AMÉRICA LATINA: EL TEMA EMBLEMÁTICO DE LAS POLÍTICAS DE RESIDUOS PELIGROSOS EN ARGENTINA EN LOS AÑOS 90
}

\author{
Jorge Gabriel Foa Torres ${ }^{1}$
}

Recibido el 28 de febrero de 2015, aprobado el 16 de junio de 2015 y actualizado el 10 de noviembre de 2015

DOI: 10.17151/luaz.2016.42.18

\section{RESUMEN}

Este trabajo presenta un análisis político-discursivo del proceso de ecologización experimentado por los mercados y Estados latinoamericanos durante la década de los 90 a través del estudio del tema emblemático de las políticas de gestión ambientalmente adecuada de residuos peligrosos en Argentina. La perspectiva en la que se inscribe este artículo es la del análisis posmarxista de políticas que busca poner énfasis en los momentos fundacionales de los procesos de significación implicados en las políticas públicas.

Para la comprensión y crítica de tal proceso de ecologización, se identificó a la lógica de la gestión ambientalmente adecuada como categoría que permitió condensar un cúmulo de prácticas discursivas orientadas a: reducir al Estado a mero gestor del mercado, autorrestringir sus atribuciones soberanas y limitar su tarea en torno a los residuos peligrosos a la función registral en pos de favorecer a las estrategias de autocontrol empresarial.

La explicación del problema de investigación construida a partir de la lógica de la gestión ambientalmente adecuada dio lugar, a su vez, a la identificación de una hipótesis explicativa central del proceso de ecologización de los años 90 en Argentina y América Latina: al desarrollo sustentable en tanto patrón de desarrollo y referencia de sentido central en la década a la hora de significar a los problemas ambientales y sus soluciones más adecuadas. La idea de patrón implica, en este caso, antes que cualquier pretendida intervención humanitaria-ecológica internacional para la protección técnica del entorno natural, la vía más adecuada para, por un lado, la radicalización de las condiciones estructuralmente heterogéneas de una economía periférica como la Argentina y, por otro, la profundización de las asimetrías entre los países del Norte y los del Sur así como también la transnacionalización del aparato productivo nacional.

\section{PALABRAS CLAVE}

Posmarxismo, políticas públicas ambientales, residuos peligrosos. 


\title{
LOGICS OF ENVIRONMENTALLY ADEQUATE MANAGEMENT AND THE SUSTAINABLE DEVELOPMENT PATTERN: THE EMBLEMATIC ISSUE OF THE HAZARDOUS WASTE POLICIES IN ARGENTINA IN THE 90's
}

\begin{abstract}
This article presents a discursive-political analysis of the greening process experienced by the markets and Latin American States during the 90's through the study of the emblematic issue of the environmentally sound management policies of hazardous waste in Argentina. The perspective of this article is the post-Marxist analysis of policies that seeks to put emphasis on the founding moments of significant processes involved in public policies.
\end{abstract}

To understand and critique such greening process, it was necessary to identify the logic of the adequate environmentally sound management as a category that allowed condensing a cluster of discursive practices aimed at: reducing the State to mere market operator, self-restrict its sovereign powers, and limit its work around hazardous waste to the information recording function towards favoring strategies of business self-control.

The explanation of the research problem constructed from the logic of the environmentally sound management led, in turn, to the identification of a central explanatory hypothesis of the greening process in the 90's in Argentina and Latin America: sustainable development as development pattern and central reference point in the decade when it comes to signify environmental problems and their most adequate solutions.

The idea of pattern implies in this case, rather than any supposed international humanitarian-ecological intervention for the technical protection of the natural environment, the most adequate, on one hand, for the radicalization of structurally heterogeneous conditions of peripheral economy such as the Argentinian economy and, on the other hand, to go deeper into the asymmetries between the North and South countries as well as the transnationalism of the national productive.

KEY WORDS: Post-Marxism, environmental public policies, hazardous waste.

\section{INTRODUCCIÓN}

Como se suele señalar desde la historia ecológica, "cada una de las formas de organización de los seres humanos tiene una peculiar manera de relacionarse con la naturaleza" (Brailovsky, 2009, p. 11). No obstante, lo ambiental ha adquirido en las últimas cuatro décadas una particular significación e importancia social. La creciente convicción de que el mundo se encuentra sometido a una crisis de alcances globales susceptible de afectar a corto o a largo plazo a todos por igual, sean países ricos o países pobres, constituye un aspecto fundacional en la historia ambiental contemporánea.

En tal contexto, este trabajo presenta un análisis político-discursivo del proceso de ecologización experimentado por los mercados y Estados latinoamericanos durante la década de los 90, a través del estudio 
del tema emblemático de las políticas de gestión ambientalmente adecuada de residuos peligrosos en Argentina.

La perspectiva en la que se inscribe este artículo es la del análisis posmarxista de políticas ${ }^{2}$. La cual abreva en diferentes tradiciones teóricas. Desde la obra marxiana a la teoría política del discurso (Laclau, 2000; Laclau y Mouffe, 2000, 2004), los aportes de la Escuela de Essex (Stavrakakis, 1999, 2007, 2009; Glynos y Howarth, 2007; Stavrakakis y Glynos, 2008) y la enseñanza freudiano-lacaniana, entre otros. Desde tal enfoque se busca poner énfasis en los momentos fundacionales de los procesos de significación implicados en las políticas públicas, es decir aquellas instancias políticas de emergencia del problema de investigación.

En tal sentido, la perspectiva exige un análisis diacrónico de los regímenes de prácticas discursivas involucrados en el campo de las políticas públicas en cuestión. En otras palabras, la historización política de esos procesos no es concebida como un esfuerzo de contextualización del problema de investigación sino como aspecto clave para su comprensión. Veamos, ya en la Primera Cumbre de la Tierra de 1972 desarrollada en Estocolmo (Suecia), el hito fundacional de la historia ambiental contemporánea, se evidenció el antagonismo y la disputa política entre países desarrollados (en adelante PD) y países en desarrollo (en adelante PED $^{3}$ ) en torno a la política ambiental internacional. Tanto fue así que el boicot del bloque soviético sumado al cuestionamiento efectuado por los PED a la legitimidad de la problemática ambiental, frente a otros problemas acuciantes para sus realidades nacionales como la pobreza y el subdesarrollo, puso en serio riesgo la realización de la Conferencia de Naciones Unidas sobre el Medio Humano ${ }^{4}$. Es decir, el antagonismo Norte-Sur es un aspecto fundacional y no secundario de la causa ambiental contemporánea.

Por caso, para Adil Najam es posible identificar tres períodos en el comportamiento de los PED para con el proyecto internacional ambiental: la postura contestataria hasta 1972, la participativa entre ese año y 1992, en que se lleva a cabo la Segunda Cumbre de la Tierra de Río de Janeiro. $\mathrm{Y}$, desde allí en adelante, identifica a la conducta comprometida con el nuevo pacto global del desarrollo sustentable. El autor considera que el paso de una posición contestataria vigorosa al compromiso se da en el marco del cambio en la forma del discurso ambiental, que pasa de denominarse "Política Ambiental Global" a "Política Global del Desarrollo Sustentable": "El desarrollo sustentable ha permitido que los países en desarrollo 'compren' la empresa ambiental global" (2005, p. 317). Asimismo, para Najam tal empresa ambiental global adquiere legitimidad para el Sur a partir específicamente del Informe Brundtland de 1987. La propuesta de Najam nos permite identificar diversas formas de hacer con ese antagonismo Norte-Sur que a lo largo de las últimas décadas ha marcado el rumbo de la historia ambiental contemporánea. Y, específicamente, a la década de los años 90 como el período de compromiso con la causa ambiental por parte de los países menos desarrollados.

El proceso de Brundtland-Río tuvo a la modernización productiva como uno de sus pilares. Orientada principalmente a los PED, es decir a aquellos que a diferencia de los más industrializados no habían alcanzado aún un desarrollo sustentable, esta modernización se intentó plasmar a través de intervenciones estatales específicas motivadas y alimentadas por la causa ambiental global y sus compromisos emergentes. En tal 
marco, frente a las protestas crecientes por el hallazgo de desechos tóxicos en África y otros países subdesarrollados, en 1989 se lleva adelante en Basilea (Suiza) la Conferencia sobre Control de los Movimientos Transfronterizos de los Desechos Peligrosos y su Eliminación, que desembocó en el Convenio del mismo nombre (Kummer, 2011) ${ }^{5}$. Este Convenio se constituyó poco tiempo después en un fuerte impulso para la expansión global de las políticas de residuos peligrosos.

En tal sentido, la Unidad Conjunta conformada a finales de los años 80 entre la Comisión Económica para América Latina y el Caribe (CEPAL) y el Programa de Naciones Unidas para el Medio Ambiente (PNUMA), junto al financiamiento y el aporte técnico de la Sociedad de Cooperación Técnica Alemana (GTZ), tendrán un rol clave en la difusión, formulación e implementación de políticas de gestión ambientalmente adecuada de residuos peligrosos en América Latina.

A nivel nacional, en los mismos inicios de la década (el 17 de diciembre de 1991) el Congreso de la Nación Argentina sancionó la Ley 24.051 (promulgada un mes después por el Poder Ejecutivo) que estableció el régimen de desechos peligrosos. Esta norma dictada durante el primer mandato presidencial de Carlos Menem, mientras ya se encontraba al frente de la Secretaría de Recursos Naturales y Desarrollo Sustentable de la Nación la ingeniera María Julia Alsogaray, tuvo el asesoramiento y participación activa de la CEPAL (HSN, 1991). Durante esta década que llamaremos "verde" tanto la ciudad de Buenos Aires primeramente ${ }^{\underline{6}}$, como luego la ciudad de Córdoba, se constituyeron en casos testigo de los proyectos ejecutados por CEPAL/PNUMA y GTZ, junto a otros Estados locales latinoamericanos ${ }^{7}$.

En tal contexto, este artículo se ordena con base en los siguientes interrogantes: ¿De qué manera es posible caracterizar el sentido de las políticas ambientales a partir del tema emblemático de los residuos peligrosos en Argentina durante los años 90? ¿Cuáles han sido las características salientes del proceso de ecologización experimentado por los mercados y Estados latinoamericanos en las últimas décadas?

En lo que sigue, se comenzará por precisar algunos aspectos ontoepistemológicos de la perspectiva propuesta y su relación con el problema de investigación. Luego, se presentará a la categoría intermedia " "lógica de la gestión ambientalmente adecuada", la cual intenta capturar a los modos en que la cooperación internacional en materia ambiental (en nuestro caso alemana vía GTZ), encauzada a través de los organismos regionales de Naciones Unidas (CEPAL y PNUMA) y las instituciones y funcionarios locales, significaron (de manera dominante) a la relación entre comercio internacional y política ambiental, y al rol del Estado y los agentes del mercado en la gestión de los residuos peligrosos.

\section{ELEMENTOS DEL ANÁLISIS POSMARXISTA DE POLÍTICAS AMBIENTALES}

Como se señaló más arriba, el análisis posmarxista de políticas reúne los aportes de diferentes tradiciones de pensamiento. No obstante, es posible sintetizar los principales fundamentos onto-epistemológicos de tal perspectiva y sus consecuencias metodológicas a la hora del análisis de políticas del siguiente modo: 
a) El punto de partido ontológico reside en el reconocimiento de la presencia de una negatividad material que impide que la realidad social se constituya de manera plena. En otras palabras, no se aborda a los procesos de significación como determinados en última instancia por referencias extradiscursivas de carácter positivo, es decir dotadas de sentidos ciertos y últimos que aseguren actual o progresivamente la plenitud de lo social ${ }^{9}$.

La máxima laclauciana de "la sociedad es imposible" (2000) apunta justamente a poner en cuestión la idea de una totalidad social en donde el sentido de todo elemento o proceso social está ya-siempre fijado por el modo en que se inserta en el sistema de relaciones. En tal línea tampoco el derecho o, para ser más precisos, la forma jurídica es idónea para suturar esa totalidad social. A partir de ello, lo estatal ya no será reducible ni al modo en que jurídicamente se autopresente ni a instancia de mera gestión administrativa de un plan ya previsto. Por el contrario, el Estado se constituye en referencia y arena privilegiada de las disputas políticas de sentido de elementos identitarios que intentan encarnar esa plenitud imposible.

b) A nivel epistemológico estos fundamentos conducen a que nuestro foco esté puesto en:

- Las prácticas sociodiscursivas, y sus regímenes, que estructuran a través de sus reglas y lazos ideológicos a diferentes espacios sociales en un tiempo determinado.

-La historización radical de los procesos significativos como directriz ineludible para comprender las instancias políticas de emergencia del problema de investigación.

- El Estado como un lugar de lo social permanentemente sobredeterminado ${ }^{10}$ por las prácticas discursivas que lo constituyen contingentemente en un determinado tiempo y espacio. $Y$ las políticas públicas, como una construcción sociodiscursiva radicalmente contingente cuya significación es el resultado de los esfuerzos y disputas políticas protagonizadas por diferentes actores sociales por sobredeterminarlas.

c) Por último, a nivel metodológico las consecuencias más destacadas son las siguientes:

- La importancia de los aspectos ontológicos, epistemológicos y teóricos en nuestra labor heurística no debe llevar a subvalorar o a generar torpes reduccionismos de nuestro problema de investigación. Por lo tanto, el principal interés estará en su explicación mediante lógicas o categorías intermedias que permitan articular los fundamentos ontológicos de la perspectiva con las exigencias epistémico-metodológicas de una investigación concreta y dar forma a la comprensión crítica de las prácticas y regímenes de prácticas sociodiscursivas implicadas en las políticas ambientales (Glynos y Howarth, 2007; Saur, 2008).

- El análisis político de fuentes documentales y entrevistas en profundidad se ordenó a través de una serie de agentes discursivos protagónicos en red relacional de producción de sentido. Preferimos esta denominación en lugar de la de variables para evitar referir a nociones inconsecuentes con nuestros fundamentos epistemológicos. De tal modo, el concepto de agentes disparadores o protagónicos para el análisis implica que los 
mismos refieren a construcciones simbólico-discursivas y de ningún modo a magnitudes más o menos matematizables. Los agentes utilizados fueron: el modo de conceptualizar a los residuos peligrosos, al rol del Estado y al de los agentes del mercado, a la función de los organismos internacionales y de cooperación y al de las organizaciones no gubernamentales en la misma, la forma en que son significados el derecho ambiental, la institucionalidad ambiental y en que es significada la riesgosidad ambiental y la contaminación ambiental.

A partir de allí se hizo posible la identificación de invariantes discursivas que, una vez reunidas, permitieron precisar a ciertos conceptos nodales que ciñen las significaciones. Luego, desde estos conceptos nodales, es posible construir a las lógicas o categorías intermedias arriba mencionadas $\underline{\underline{11}}$.

- La utilización de la estrategia del tema emblemático para el estudio de políticas ambientales (Hajer, 2005) permite, en el contexto de los numerosos aspectos y tópicos ambientales, identificar aquel que en cierto tiempo y espacio puede convertirse en el asunto ambiental clave para ciertas instituciones y sus políticas, señalando el camino para la caracterización y crítica de los modos en que los problemas ambientales son concebidos, la manera en que las soluciones al mismo son delineadas y las formas en que los cambios político-institucionales son planeados y ejecutados para hacerles frente.

- La centralidad del problema de investigación, en tanto objeto destinado a ser paulatinamente deconstruido en aras de precisar sus fundamentos radicalmente políticos. En tal sentido, las hipótesis se ubican en el marco del proceso retroductivo de explicación como una instancia de inferencia que no emerge hasta que su contenido no está ya presente en la explicación misma del problema que se aborda (Glynos y Howarth, 2007).

Ahora bien, la indagación articulatoria que permanentemente llevó del caso estudiado y el tema elegido hacia sus diferentes contextos discursivos es casi imposible de ser traducida respetando fielmente el derrotero seguido en la tarea investigativa. Por lo que en este artículo se presentarán a continuación los componentes conceptuales más destacados de la categoría lógica de la gestión ambientalmente adecuada, construida a los fines de condesar el cúmulo de prácticas discursivas en juego. Asimismo, en el transcurso de la indagación se advirtió la importancia del plano internacional al momento de sobredeterminar el sentido de las condiciones políticas de emergencia del sintagma gestión ambientalmente adecuada en América Latina $\mathrm{y}$, específicamente, Argentina.

Por tales motivos, cabe aclarar que no se propondrá una descripción densa de hechos o citas textuales de fuentes ${ }^{12}$ sino los ejes del análisis crítico articulado con base en tal lógica y sus significantes nodales. A tales fines, se comenzará por dar cuenta del contexto regional de emergencia y de sus aspectos más relevantes. Luego se pondrá el foco en el nivel nacional. Cabe hacer notar, asimismo, que a partir de términos o sintagmas naturalizados como el de "gestión ambientalmente adecuada" y temas sedimentados como puramente técnicos como el de las políticas públicas ambientales de residuos, fue posible desandar una compleja trama discursiva siempre orientados por el deseo de historizar políticamente al problema de investigación. 
A continuación, se comenzará por abordar a un elemento sustantivo del proceso de ecologización de los mercados y Estados latinoamericanos: la expansión hacia nuestra región de la denominada "industria del ambiente". Éste y los demás aspectos que se abordarán son centrales a la hora de comprender el sentido de "lo ambientalmente adecuado" de la gestión que, en Argentina, se desplegó a través de las políticas de residuos peligrosos.

\section{INDUSTRIA DEL AMBIENTE Y LÓGICA DE LA GESTIÓN AMBIENTALMENTE ADECUADA EN AMÉRICA LATINA}

El desarrollo exponencial a escala global de la industria del ambiente durante la década de los años 90 permitió que sea ubicada por algunos al mismo nivel de la industria aeroespacial y de la farmacéutica (Ruz y Mladinic, 2005). A principios de la década el volumen total de la industria se estimaba en el orden de los 250 mil millones de dólares (OECD, 1993), en los 2000 el monto ascendía a US $\$ 450$ mil millones y el proyectado para el 2010 era de US\$640 mil millones (Ruz y Mladinic, 2005).

La definición de cuáles actividades deben ser incluidas dentro de la noción de industria del ambiente ha sido una cuestión arduamente discutida (Barton, 1997 $)^{13}$. Siguiendo a Barton (1997), podemos afirmar que hablar de industria del ambiente no equivale a hacer referencia a cualquier pretendida "industria ambiental" que implicare efectos ambientalmente positivos de por sí, sino tan solo un campo de la economía mundial capaz de movilizar inmensas sumas de dinero y de responder a determinadas reglas de funcionamiento, entre las cuales se encuentran aspectos estructurales del vínculo comercial-ambiental entre los países del Norte y los países del Sur.

Pero además de ello, desde nuestro punto de vista la industria del ambiente puede ser entendida en sentido amplio y no como mero sector industrial. Sino en tanto meta-sector o instancia transversal que no solo refiere a tales o cuales empresas de prestación de servicios ambientales o de producción de bienes y tecnologías verdes, sino al completo proceso de ecologización del modo de producción industrial capitalista emergente de la instauración del patrón de desarrollo sustentable.

Hablar de la emergencia y desarrollo de la industria del ambiente no implica necesariamente referir a un pretendido progreso libre del sector ambiental del mercado. Por el contrario, la consistente intervención temprana de ciertos Estados altamente industrializados, para ofrecer respuestas de mercado a la "inseguridad ambiental", los posicionó como pioneros o first movers en la industria del ambiente global (específicamente en los 90: EE.UU., Alemania y Japón). En este contexto la lógica de la gestión ambientalmente adecuada en América Latina, promovida por organismos internacionales, multilaterales y por la cooperación internacional, identificó a los first movers como merecedores, en función de su conducta ambientalmente responsable, de las ventajas provenientes de las nuevas reglas verdes del mercado internacional (Barton, 1997).

La expansión de la industria del ambiente (en el sentido amplio que se propuso más arriba) hacia Latinoamérica encontró su sostén en la idea de la necesaria puesta en acción de tres tipos de transferencia desde los PD hacia los PED. Por un lado, la transferencia de la conciencia de los riesgos 
y peligros ambientales adquirida por los consumidores del Norte a través de la labor de ONG ambientalistas y la formación de técnicos sensibilizados con la causa ambiental. Por otro, la transferencia del moderno derecho ambiental, y la universalización de sus principios generales fundados básicamente en la idea de prevención de esos riesgos ambientales, mediante la promoción de la creación de instituciones y regulaciones propiamente ambientales en la región. Por último, la transferencia tecnológica de los bienes de capital y know how verde a través de la liberalización del comercio internacional $1^{\underline{14}}$.

Pero tal proceso fue ordenado a través de nuestro método retroductivo mediante la identificación de la lógica de la gestión ambientalmente adecuada, en tanto forma dominante de significar a los problemas ambientales y sus soluciones posibles que se constituyó en la década de los 90 en una tecnología gubernamenta ${ }^{15}$ en materia ambiental. En América Latina esta lógica se articuló en torno a una serie de puntos nodales que se desarrollan a continuación.

\section{La centralidad de los requerimientos ambientales de los consumidores y países del Norte}

Hacia principios de los años 90 y durante toda esa década, la conciencia ambiental desarrollada por los ciudadano-consumidores de los países del Norte, será visualizada, como una poderosa fuerza de mercado capaz de arrastrar a esos Estados al establecimiento de requerimientos ambientales que limiten el ingreso o importación de productos, que en sí mismos o en virtud de las características del proceso productivo del que son resultado, impliquen un riesgo ambiental intolerable para sus territorios o para el equilibrio ecológico del planeta.

Los requerimientos ambientales suelen constituirse en requisitos o exigencias que determinados países en ejercicio de su soberanía, y aún en violación de acuerdos comerciales internacionales, pueden imponer tanto a productos como a procesos productivos de otros países. Si bien se ha reconocido que los mismos suelen convertirse en barreras paraarancelarias internacionalmente ilegales, también se ha destacado su tendencial carácter forzoso e, incluso, sus beneficios en materia ecológica ${ }^{\underline{16}}$.

Cabe advertir que mientras el ejercicio de soberanía por parte de los PD es legitimado a partir de sus motivaciones ambientales, es notoria la ausencia en este discurso de referencias a la soberanía de los países receptores de tales políticas comerciales. Antes bien, es supuesta una posición pasiva de los países latinoamericanos orientada al aprendizaje de la experiencia de los PD y a la adaptación sin ambages frente a las nuevas condiciones ambientales del mercado internacional.

De tal modo, los requerimientos ambientales de los $\mathrm{PD}$, antes que simples esfuerzos por la protección desinteresada del ambiente global, lograron constituirse en verdaderos patrones fácticos de desarrollo que los agentes económicos de los PED debían considerar con suma atención si pretendían acceder a los mercados de los países del Norte ${ }^{\underline{17}}$.

\section{¿Dónde están los paraísos de contaminación?}

Hacia inicios de los 90 el discurso ambientalista sostenía la concepción por la cual la apertura económica indiscriminada en países periféricos 
traería como consecuencia la instalación en los mismos de las industrias sucias provenientes de los PD. Estas industrias estarían de ese modo en condiciones de aprovechar las ventajas en términos de costos provistas por las debilidades jurídico-institucionales en materia ambiental de los países Latinoamericanos. En ese marco es que surge la noción de "paraísos de contaminación" o pollution heavens, para hacer referencia a los países en donde las ventajas competitivas respecto de aquellos que poseen regulaciones ambientales más desarrolladas, darían paso a la radicación de actividades productivas altamente contaminantes y carentes de los controles y mecanismos de producción promovidos en los PD.

Pero rápidamente diferentes organismos, entre los que se destaca el Banco Mundial, salieron al cruce de esta concepción. Un documento clave en esta discusión es el artículo "Política comercial y contaminación industrial en América Latina: ¿Dónde están los paraísos de contaminación?" 18 , publicado en 1993 en el Journal of Environment and Development. Sus autores Nancy Birdsall y David Wheeler se desempeñaban por aquel entonces, como Directora y Economista Senior, respectivamente, del Departamento de Economía del Banco Mundial. A partir de su publicación el artículo será una referencia cuasi obligada al hablar de protección ambiental y comercio internacional en América Latina y fuertemente en el relato internacional del proceso de ecologización global de los mercados ${ }^{\underline{19}}$.

La propuesta clave del artículo fue una nueva forma de ubicar, entender y combatir a la amenaza de los paraísos de contaminación. Es que para Birdsall y Wheeler éstos solo pueden emerger en el marco de países sujetos a políticas económicas proteccionistas:

A partir de estudios de caso y evidencia econométrica, llegamos a la conclusión de que las economías protegidas son más propensas a favorecer industrias de contaminación intensiva, mientras que la apertura en realidad fomenta una industria más limpia a través de la importación de los estándares de contaminación de los países desarrollados. (Birdsall y Wheeler, 1993, p. 137)

Como consecuencia, las políticas de apertura comercial para los países latinoamericanos son anunciadas como medidas ambientalmente beneficiosas desde el momento en que los pollution heavens no se encuentran donde se los suele buscar, es decir en economías discrecionalmente liberalizadas, sino en Estados preocupados por la protección de sus industrias nacionales.

De tal modo se, en un primer momento, disputó y, luego, hegemonizó el sentido de los paraísos de contaminación vinculándolos a países dotados de políticas intervencionistas y proteccionistas. En tal sentido, y por otro lado, el caso de estudio fue Chile que comenzará, de este modo, a constituirse en el modelo latinoamericano de gestión ambiental ${ }^{20}$.

\section{La construcción del modelo chileno de Gestión Ambiental}

Para Andrew Hurrell, la crisis ecológica ha jaqueado la legitimidad de los Estados y, a partir del Informe Brundtland, parece emerger el consenso de que sería ineficaz abordar la cuestión con base en un sistema internacional fragmentado o perdiendo de vista la creciente debilidad de los Estados para gestionar con éxito las problemáticas ambientales 
(Hurrel, 1994). Este espíritu globalizador de la década verde fue indudablemente influido por la caída del muro de Berlín y la desarticulación del bloque soviético, ocurridas ambas en sus inicios. La importancia de estos acontecimientos para la causa ambiental global fue decisiva.

Como se señalo, la Primera Cumbre de la Tierra sufrió lo que se denominó como el "boicot" del bloque soviético. Esta tentativa por disputar y frustrar la causa ambiental norteamericano-europea no será olvidada ni pasada por alto. A la par de la construcción del ideal del desarrollo sustentable y de la identificación de los países del mundo que han logrado alcanzar el ideal, fue señalado el contra-modelo ambiental global: el bloque soviético con sus enormes pasivos ambientales ${ }^{21}$. El modelo socialista en su conjunto fue de tal modo identificado como contra-modelo de gestión ambiental y, al mismo tiempo, corrieron con la misma suerte las políticas intervencionistas y planificadoras del Estado.

En la construcción del modelo chileno, por su parte, el objetivo estuvo puesto en refutar que la liberalización económica arrastre a los países a convertirse en paraísos de contaminación. Por el contrario, los sectores industriales más contaminantes tenderían a expandirse menos y los más limpios a desarrollarse $\mathrm{Li2}^{\underline{2}}$.

Se erigió, de tal modo, el modelo chileno de gestión ambientalmente adecuada en virtud tanto de su temprana adopción del patrón ambiental de desarrollo como de sus propias condiciones de emergencia histórica. El mismo Muñoz, citando a Birdsall y Wheeler, llegará a sostener que "la apertura externa en Chile [...] acelera la inversión en tecnologías que tienden a ser limpias e importadas mayormente de países con normas ambientales más elevadas" (1995, pp. 377-378).

\section{La primacía de las Corporaciones Transnacionales}

Hacia mediados de la década verde se vio ampliamente difundida, entre gobiernos y analistas, la idea de que las corporaciones transnacionales serían los vehículos más adecuados para la reducción de la brecha tecnológica entre el Sur dependiente y el Norte desarrollado (Muñoz, 1995; Chudnovsky y López, 2003). Esto en base a la identificación de las políticas proteccionistas de industrialización por sustitución de importaciones desarrolladas por los procesos nacional-populares latinoamericanos con anterioridad a las dictaduras militares y regímenes neoliberales como el posible terreno propicio para la instauración de paraísos de contaminación en América Latina (Birdsall y Wheeler, 1993).

En ese marco, la primacía de esas corporaciones es construida en el discurso internacional sobre comercio y medio ambiente en América Latina durante la década verde a partir de dos operaciones. Primeramente la identificación de ambos conglomerados de agentes económicos, corporaciones transnacionales y Pequeñas y Medianas Empresas (en adelante PYMES), haciendo énfasis en la posición más ventajosa de las primeras para mejorar la calidad ambiental de las actividades productivas del país en el que eventualmente se radicasen ${ }^{23}$. En segundo lugar, el establecimiento de un específico modo de vinculación entre ambas: el de la libre competencia desprovista de la intervención proteccionista de los Estados. Es que, toda clase de políticas proteccionistas promovería bajo esta concepción la ampliación de la brecha tecno-ecológica ${ }^{24}$. 
De tal modo, la promoción de la expansión global de la industria del ambiente se inscribió en un paulatino proceso de consolidación del desarrollo sustentable como patrón o modelo de tenor fundamentalmente tecnológico-comercial. La exclusión de la posibilidad de valerse del dumping ecológico ${ }^{25}$ entre el espectro de alternativas políticas soberanas en Latinoamérica (susceptibles de haber encauzado de otro modo sus modos de producción y distribución del excedente) se constituyó en un elemento clave de la lógica de la gestión ambientalmente adecuada.

\section{LA LÓGICA DE LA GESTIÓN AMBIENTALMENTE ADECUADA EN ARGENTINA EN LOS 90}

En el marco de las políticas públicas ambientales en Argentina en los años 90 , las referidas a residuos peligrosos adquirieron un lugar de gran importancia siendo, como se vio anteriormente, la primera ley de política ambiental promulgada durante el gobierno de Carlos Menem en el año 1992.

Ahora bien, desde nuestra perspectiva, su importancia estriba, en primer lugar y como se vio en el apartado anterior, en haberse constituido en un tema que, inscripta en la gestión ambientalmente adecuada, articuló en la problemática del comercio internacional con el de la protección ambiental. En segundo, que la temática involucra a la regulación de gran parte del sistema productivo de un Estado a través de la instauración de ciertos criterios para la valoración de la riesgosidad de sus diversos sectores productivos. De tal modo, las políticas se constituyeron en tema relevante y emblemático de las políticas ambientales de la década a nivel nacional.

En tal marco nuestra categoría de análisis, lógica de la gestión ambientalmente adecuada, también permite identificar a los aspectos más destacadas del proceso de políticas públicas de residuos peligrosos en Argentina. Aspectos que se abordarán a continuación.

\section{Experiencia internacional y autointerdicción}

La interdicción refiere a aquella figura jurídica basada en la necesidad de asegurar la protección de una persona que carece de la aptitud suficiente para ejercer sus derechos y contraer obligaciones. No obstante, a lo largo de la historia, el instituto no solo fue pensado en términos protectivos sino también como sanción o pena adicional para los penalmente condenados. En cualquier caso, lo que nos interesa rescatar es la noción de interdicción en tanto cercenamiento en el ejercicio de ciertos derechos. $Y$ específicamente en el caso de nuestro análisis, la abdicación por parte del Estado a ejercer el derecho al desarrollo soberano a través del sometimiento de sus estructuras gubernamentales al imperativo transnacional del desarrollo sustentable.

Pero pasemos a apreciar de qué manera estos conceptos emergen de las prácticas discursivas en torno a las políticas de gestión ambientalmente adecuada de residuos peligrosos en Argentina.

El por entonces Subsecretario de Medio Ambiente de la Municipalidad de la Ciudad de Buenos Aires, el biólogo Alberto Morán, afirmaba en ocasión de la reunión de trabajo sobre residuos tóxicos (CEPAL-Senado de la Nación) inmediatamente anterior a la sanción de la Ley Nacional 24.051: 
[...] agradezco mucho el apoyo de la CEPAL porque para nosotros es sumamente importante ya que no sabemos formular políticas y necesitamos ayuda, no sabemos bajar esas políticas a la realidad, no conocemos estrategias concretas de transformación de ésta. Tampoco tenemos toda la experiencia necesaria para obtener los recursos como para poder hacer esto. (HSN, 1991, p. 3543, énfasis agregado)

Tales afirmaciones no implicaron simplemente a un reconocimiento del desconocimiento o la impericia para formular políticas ambientales vernáculas sino, más específicamente, a la renuncia gubernamental de considerar un rol del Estado diferente al establecido por una específica e históricamente situada lógica política: la de la gestión ambientalmente adecuada. $Y$ tal rol implicaba un posicionamiento pasivo y de sometimiento al imperativo de la causa ambiental global que contuvo, al menos, dos tipos de abdicación. Por un lado, la renuncia a poner en cuestión el mismísimo mandato por incorporar lo ambiental a las estrategias de desarrollo nacional. Y, por otro, a la búsqueda de alternativas propiamente nacionales a las modalidades propuestas desde el contexto internacional ${ }^{26}$.

De tal modo, la prioridad no estuvo puesta en la construcción de lineamientos o criterios nacionales capaces de tener en cuenta las necesidades y particularidades de este territorio, sino en la experiencia internacional, en tanto saber más adecuado o apto para la formulación de políticas ambientales.

En ese contexto, el rol de la unidad conjunta CEPAL/PNUMA en el proceso de sanción de la ley nacional como en la gestión municipal nos permite ubicarla como principal vía de canalización hacia Argentina de las políticas de gestión ambientalmente adecuada de residuos peligrosos. Canalización que no podría haberse llevado a cabo de la manera en que se llevó adelante sin esta suerte de autointerdicción de las autoridades gubernamentales.

Este marco institucional internacional de Naciones Unidas sirvió para que la cooperación internacional de Alemania, uno de los países pioneros en la industria ambiental, tuviera un destacado rol. A través de la organización de visitas a ese país y capacitaciones para expertos y funcionarios locales, se convirtió en una referencia obligada a la hora de hablar de gestión ambientalmente adecuada.

\section{Las políticas de autocontrol}

El avance del sector privado en la gestión ambiental a través del establecimiento de mecanismos de autocontrol empresarial ${ }^{27}$, fue un objetivo central de la política ambiental en Argentina en los $90^{\underline{28}}$. La ingeniera Alsogaray precisaba del siguiente modo al rol del Estado frente en el sostenimiento de las políticas de autocontrol:

[...] la empresa actúa casi como un delegado del Estado al ejercer, de algún modo, el poder de policía. A su vez, la entidad certificante es la que controla. Con lo cual, el Estado ahorra inspectores y evita problemas de competencia y posibles centros de corrupción. La transparencia de este sistema resultaría muy costosa para el Estado si pretendiera reproducirla. (1998, p. 9) 
Las menciones acerca de la independencia del "poder político" y la "transparencia" del sector privado en contraposición con la corrupción del Estado no deben llevarnos a pensar únicamente en cierta moral capitalista-neoliberal de este relato. Por nuestra parte lo que nos interesa es destacarlos como estrategias retóricas y argumentativas orientadas a sustraer del espacio de la planificación estatal a todo un extenso campo de la vida socioeconómica de cualquier comunidad contemporánea: de los desechos de sus actividades industriales.

Pero esta concepción del rol del Estado, que llamaremos función registral, ya estaba presente en palabras de los funcionarios de CEPAL participantes del proceso de sanción de la Ley 24.051. Es decir, se inscribía en procesos más amplios de significación de las decisiones de economía política que afectaban a la región ${ }^{29}$.

Hablar de la reducción de la tarea estatal a la función registral no implica pensar sencillamente en términos de un "Estado ausente" sino en un Estado fuertemente presente en aquellas instancias que hacen posible la autogestión del sector privado. El Estado, en definitiva se ubica como un agente del mercado encargado, a su vez, de dotarlo de legalidad y legitimidad pública oficial.

En realidad, el autocontrol no es más que un eufemismo que vela al imperativo de la estandarización y la homogeneización para la transnacionalización de la economía nacional. Es que lo que se juega en este aspecto de las políticas autocontrol es el escaso o nulo apoyo y financiamiento estatal a la investigación y el desarrollo tecnológico nacional. Este es otro elemento nodal de la lógica de la gestión ambientalmente adecuada $\mathrm{y}$, particularmente, de su auge en Argentina durante la década verde ${ }^{30}$.

\section{¿Un paraíso de tecnologías limpias?}

Los paraísos de contaminación debían ser buscados, para la concepción dominante instaurada por el Banco Mundial, en las economías proteccionistas antes que en las abiertas o liberalizadas a los flujos y reglas del mercado internacional. Esta tesis también fue sostenida en un sentido similar en las instancias fundacionales de la política nacional de desechos peligrosos, tal cual afirmaba el Diputado Justicialista Oscar Alfredo Blanco promotor de la Ley 24.051 ante la Cámara: "Los países subdesarrollados que como el nuestro padecen estas falencias tienden a convertirse en paraísos de contaminación, que al estilo de los famosos paraísos fiscales dan involuntaria visa de entrada a todo tipo de residuos peligrosos" (HCDN, 1991, p. 3349).

La profusión de tratados internacionales y normativas nacionales se sumó al proceso de estandarización y transnacionalización de las economías en pos del objetivo de la sustentabilidad. La declinación del poder de los Estados encuentra de esa manera su legitimación en los supuestos beneficios ambientales de las reglas verdes del mercado. De tal modo, el sector público debe ceder en su intervención a los fines de evitar cualquier tipo de distorsión que ponga en riesgo la plena aplicación de esas reglas. En otras palabras, el Estado bajo esta concepción deberá hacer todo lo que esté a su alcance para insertar al aparato industrial del país en el marco normativo transnaciona $\left.\right|^{31}$. 
Pero para que un Estado se someta al imperativo ecológico transnacional no es suficiente que dicte normas y establezca instituciones concordantes con los patrones jurídico-administrativos establecidos por organismos y actores internacionales. Es decir, poco serviría a la causa ambiental global que el país se limitara a sancionar normas inspiradas en los lineamientos verdes internacionales si luego se establecieran políticas de protección de la industria local y/o de las pequeñas y medianas empresas. El requisito indispensable de tal ecologización implicada en la lógica de la gestión ambientalmente adecuada fue la apertura económica indiscriminada.

\section{Proceso de ecologización y modelo de acumulación}

Siguiendo a Eduardo Basualdo (2011), hacia el año 1976 el modelo de acumulación y producción en Argentina estaba centrado en la industrialización por sustitución de importaciones. Sin embargo, el genocidio y el terror impuesto por la dictadura militar se constituyeron en la condición de posibilidad para la transformación estructural de la economía Argentina. A partir de allí, la imposición del modelo de valorización financiera encontró su fundamento en el desarrollo y expansión de los mercados financieros a nivel internacional. Por esos años, las importantes inversiones en innovaciones técnicas dieron lugar a una revolución tecnológica que provocó modificaciones radicales en los modos de producción, acumulación y transferencia del excedente en los países centrales y, luego, en los periféricos ${ }^{32}$.

La recuperación de la democracia en Argentina con la presidencia de Raúl Alfonsín, enfrentó una muy difícil transición con la sombra de la amenaza militar aún en ciernes. La puja entre sectores económicos y entre éstos y el Gobierno condujo a una crisis económica de enorme envergadura que derivó en la salida anticipada de Alfonsín y la asunción del dirigente peronista riojano Carlos Menem.

A partir de allí se consolidaría, en términos de Basualdo, la "revancha clasista" contra los sectores populares encarnada por parte de la oligarquía argentina y sectores del capital financiero internacional. Es en este contexto que adquiere auge el proceso de ecologización implicado en la lógica de la gestión ambientalmente adecuada, constituyéndose en una fuerza legitimatoria nodal para la consolidación del modelo de acumulación financiera instaurado con la dictadura militar.

El eje de esa revancha clasista fue la desarticulación del proyecto de industrialización sustitutiva. La enorme crisis en la que deriva el país hacia finales de los años 90 con un desempleo en niveles inéditos en consonancia con una retracción también histórica de la actividad industrial se dieron "como resultado de una mayor concentración de capitales, progreso técnico y poder económico en grupos privilegiados de empresas nacionales y extranjeras" (Salvia, 2012, p. 382).

Por lo tanto, si el crecimiento económico de los primeros años de la década "verde" en Argentina no obedeció al desarrollo de las fuerzas productivas sino a la acumulación de capital fijo en consonancia con el modelo de apreciación financiera, sus impactos no golpearon en una tabla rasa sino que profundizaron las condiciones estructuralmente heterogéneas y desiguales de la economía argentina (Salvia, 2012). Y tal escenario no fue el resultado de condiciones técnicas y requerimientos ambientales neutrales del mercado internacional sino de decisiones gubernamentales que posicionaron al Estado en la autointerdicción y la renuncia a su soberanía frente a los procesos políticos que por aquellos 
años se vivían. En tal sentido, a continuación se abordará críticamente al principio del derecho ambiental internacional "el que contamina paga", ampliamente expandido en América Latina y Argentina a partir de la década de los 90 y su impacto en la economía de un país periférico.

\section{El que contamina paga o la selección de los perdedores del proceso de ecologización}

La lógica de la gestión ambientalmente adecuada no solo se orientó al establecimiento del mercado de los residuos peligrosos. Si hubiésemos reducido nuestra mirada a este punto, nos hubiésemos privado de echar luz a otros órdenes de consecuencias del proceso de ecologización. El efecto legitimador de la construcción del riesgo del desarrollo industrial argentino en particular, claramente desborda el ámbito del mercado de los residuos peligrosos. En un vector que va desde los residuos de la producción a las condiciones estructurales de la economía nacional, el proceso de ecologización de los mercados y Estados latinoamericanos durante la década verde es indistinguible de sus implicancias en términos geopolíticos, de especialización productiva y sometimiento a los imperativos de la división del trabajo internacional.

Pero cuando se habla del riesgo del desarrollo industrial argentino no se hace refencia al conjunto de los sectores productivos. Evidentemente durante los años 90 hubo un gran desarrollo de aquellas ramas de mayor productividad y muchas de ellas pasaron también por procesos de integración compleja en cadenas transnacionales de producción. El objetivo aquí no pasa por desagregar sector por sector, actividad por actividad y comparar cuantitativamente sus desempeños. A lo que se apunta es a la íntima vinculación entre política ambiental y política económica, entre construcción social del peligro ambiental y modelo de acumulación, entre los escenarios de concientización-legitimación ciudadana y el modo de producción y distribución del excedente que se busca imponer o sostener.

En definitiva, lo que interesa poner de relieve es que el proceso de ecologización en su matriz político-conceptual no es igual a la sumatoria o a las diferencias de desarrollo sector por sector, sino a la legitimación ambiental de políticas orientadas a la especialización productiva, desindustrialización y profundización de las desigualdades estructurales de una economía dependiente como la Argentina.

Veamos, en el contexto argentino el principio "el que contamina paga" ${ }^{\text {"33 }}$ también fue decisivo a la hora del establecimiento de las políticas de residuos peligrosos. Esta norma era vista con muy buenos ojos y citada como ejemplo del moderno derecho ambiental que ya no solo pronunciaba meras declaraciones de buena voluntad u objetivos generales y difusos sino que era capaz de poner en acto la tan mentada protección ambiental. Lo ambientalmente adecuado exigía que, sin discriminación, cada empresa se tuviese que hacer cargo de los costos que implicaba ser identificado como posible contaminador. Aunque esos costos y esa adecuación normativa no implican necesariamente que se logre de por sí la prevención de daños ambientales.

En realidad, el principio contaminador-pagador mientras afirma que no discrimina entre contaminadores ("todo contaminador debe asumir los costos de sus actividades") supone, al mismo tiempo, al mercado como una tabla rasa en donde se desempeñan situaciones de competencia 
perfecta entre agentes económicos. Esta mirada en el mejor de los casos ingenua y, en el peor, cínica, aparenta desconocer al mercado como un terreno de posiciones de poder establecidas con anterioridad al proceso de ecologización, y sobre las cuales el derecho ambiental se declara neutral. Pero esa neutralidad no es más que el impulso para que los mejor posicionados consoliden y expandan su poder. Es decir, la cuestión sencillamente pasa por el establecimiento de reglas de juego claras para la concentración y centralización del capital en manos de los actores transnacionales y las empresas de capitales argentinos de mayor productividad.

Es a partir de todo ello que se puede afirmar que la aplicación ingenua o cínica del principio el que contamina paga constituyó bajo el predominio del modelo de acumulación por apreciación financiera, un mecanismo destacado en la profundización de la desigualdad propia de la estructuralmente heterogénea economía argentina. Es decir, en un contexto de extrema liberalización de la economía Argentina (que incluyó a la desregulación de la inversión extranjera directa) las nuevas reglas ecológicas del mercado internacional impactaron de lleno en todos los agentes económicos nacionales. Pero con mayor fuerza lo hicieron sobre aquellos de menor productividad y dificultoso acceso al financiamiento que usualmente destinan su producción al mercado interno. Específicamente gran parte de las PYMEs, economías regionales y agentes de la economía social se vieron forzados a competir en igualdad de condiciones con productos provenientes de otras latitudes y, al mismo tiempo, con escasas posibilidades de acceder a un mercado internacional en el que debían hacer frente a los costos ambientales (por ejemplo aquellos implicados en la certificación de la serie ISO 14.000). Lo cual abrió paso a que muchas de esas unidades productivas locales y nacionales pudiesen ser absorbidas por agentes transnacionales de mayor productividad y acceso al financiamiento necesario para hacer frente a esos costos ambientales.

De tal modo, lo que Piritta Sorsa (1995) anunciaba en el primer lustro de la década verde como el cambio en los patrones de producción y comercio que seguramente provocarían las quejas de las "voces de los perdedores", implica en realidad a la decisión política de otorgar primacía a las corporaciones transnacionales y los sectores industriales de mayor productividad en la conducción y control de la política y la planificación socioambiental.

\section{CONSIDERACIONES FINALES}

La lógica de la gestión ambientalmente adecuada, construida a partir de

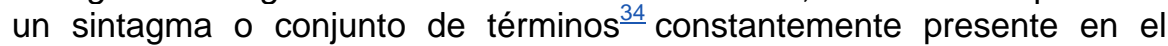
referente empírico, permitió condensar un cúmulo de prácticas discursivas orientadas a sobredeterminar el significado de las políticas públicas ambientales de residuos peligrosos en Argentina. Implicó a la reducción del Estado a mero gestor del mercado susceptible de autorrestringir sus atribuciones soberanas, limitando su tarea en torno a los residuos peligrosos a la función registral en pos de favorecer a las estrategias de autocontrol empresarial. Esta modalidad de intervención estatal, antes que una injerencia neutral como garante de un juego cuyo resultado final se ignora, dio lugar a cierta modalidad de aplicación del imperativo "el que contamina paga", que posibilitó la exclusión en Argentina durante la década verde de los agentes del mercado menos aptos para hacer frente 
a los nuevos requerimientos y reglas verdes del patrón de desarrollo sustentable.

Aquello que los ejecutivos del Banco Mundial, Nancy Birdsall y David Wheeler, postulaban en los albores de la década verde como condiciones para la transferencia y cooperación tecnológica hacia la región constituyó una disputa política en torno al control de las políticas económicas de los países de la región.

El análisis posmarxista desplegado permitió echar luz sobre las instancias políticas y por tanto contingentes del proceso de ecologización. En un vector que ya no necesariamente funge a los desechos peligrosos con la estandarización y transnacionalización de los modos de producción.

A partir de la comprensión de nuestro problema de investigación posibilitada por la identificación de la lógica de la gestión ambientalmente adecuada, emerge la hipótesis explicativa del problema de investigación planteado ${ }^{35}$ : el desarrollo sustentable en tanto patrón de desarrollo fue la referencia de sentido central a la hora de significar a los problemas ambientales y sus soluciones más adecuadas. La idea de patrón implica, en este caso, antes que cualquier pretendida intervención humanitariaecológica internacional para la protección técnica al entorno natural, la vía más adecuada para, por un lado, la radicalización de las condiciones estructuralmente heterogéneas de una economía periférica como la Argentina y, por otro, la profundización de las asimetrías entre los países del Norte y los del Sur así como también la transnacionalización del aparato productivo local y nacional.

En definitiva, el análisis posmarxista de políticas públicas ambientales permitió establecer relaciones de sentido entre elementos antes desvinculados. Una de las más destacadas reside en la estrecha vinculación entre el patrón de desarrollo sustentable, sustentado en la gestión ambientalmente adecuada, y el proceso de consolidación durante la década verde del modelo de acumulación por apreciación financiera instaurado en Argentina a partir de la dictadura militar de 1976. De tal manera, antes que cualquier pretendido paraíso de tecnologías limpias o de prevención técnica de la contaminación, el patrón se constituyó en condición de posibilidad para la centralización del capital en los sectores más concentrados y en corporaciones transnacionales.

\section{AGRADECIMIENTOS}

El autor agradece los comentarios y correcciones de uno de los evaluadores anónimos que enriquecieron y contribuyeron a precisar ciertos aspectos de este trabajo.

\section{POTENCIAL CONFLICTO DE INTERESES}

No hay. 


\section{FUENTES DE FINANCIACIÓN}

CONICET, Universidad Nacional de Villa María.

\section{REFERENCIAS}

- Barton, J. (1997). The North-South Dimension of the Environment and Cleaner Technology Industries. Discussion Paper Series. Maastricht: Institute for New Technologies of the United Nations University.

- Basualdo, E. (2011). Modelo de acumulación y sistema político en la Argentina. Notas sobre el transformismo argentino durante la valorización financiera. En Sistema político y modelo de acumulación (pp. 41-121). Buenos Aires: Cara o Ceca.

- Brailovsky, E. (2009). Historia Ecológica de Iberoamérica. II. Buenos Aires: Capital Intelectual.

- Clapp, J. (1998). The Privatization of Global Environmental Governance: ISO 14.000 and the Developing World. Global Governance, 4, 295-316.

- Foa Torres, J. (2014). Lógica del riesgo y patrón de desarrollo sustentable en América Latina: políticas de gestión ambientalmente adecuada de residuos peligrosos en la ciudad de Córdoba. Tesis Doctoral inédita. Centro de Estudios Avanzados, Universidad Nacional de Córdoba.

- Foucault, Michel (2008). Tecnologías del yo. Buenos Aires: Paidós.

- Foucault, Michel (2012). Nacimiento de la biopolítica. Buenos Aires: Fondo de Cultura Económica.

- Glynos, J. y Howarth, D. (2007). Logics of Critical Explanation in Social and Political Theory. U.K.: Routledge.

- Hajer, M. (2005). Coalitions, Practices, and Meaning in Environmental politics: From Acid Rain to BSE. En Howarth, D. y Torfing, J. (Eds.), Discourse Theory in European Politics (pp. 297315). Reino Unido: Palgrave.

- Hurrell, A. (1994). A Crisis of Ecological Viability? Global Environmental Change and the Nation State. Political Studies, XLII, 146-165.

- Laclau, E. (2000). La imposibilidad de la sociedad. En Nuevas reflexiones sobre la revolución de nuestro tiempo (pp. 103-106). Buenos Aires: Nueva Visión.

- Laclau, E. y Mouffe, C. (2000). Posmarxismo sin pedidos de disculpas. En Nuevas reflexiones sobre la revolución de nuestro tiempo. Buenos Aires: Nueva Visión. 
- Laclau, E. y Mouffe, C. (2004). Hegemonía y estrategia socialista. Hacia una radicalización de la democracia. Buenos Aires: Fondo de Cultura Económica.

- Najam, A. (2005). Developing Countries and Global Environmental Governance: From Contestation to Participation to Engagement. International Environmental Agreements, 5, 303321.

- Peralta Ramos, M. (2007). La economía política argentina: poder y clases sociales. Buenos Aires: Fondo de Cultura Económica.

- Salvia, Agustín (2012). La trampa neoliberal. Un estudio sobre los cambios en la heterogeneidad estructural y la distribución del ingreso en la Argentina: 1990-2003. Buenos Aires: Eudeba.

- Saur, D. (2008). Categorías intermedias y producción de conocimiento. En Da Porta, E. y Saur, D. (Coords.), Giros teóricos en las Ciencias Sociales y Humanidades (pp. 63-71). Córdoba: Comunicarte.

- Stavrakakis, Y. (1999). Fantasía verde y lo Real de la naturaleza: elementos de una crítica lacaniana. Tópicos de Educación Ambiental, 1(1), 47-58.

- Stavrakakis, Y. (2007). Lacan y lo político. Bs. As.: Prometeo.

- Stavrakakis, Y. (2009). La izquierda lacaniana. Bs. As.: Fondo de Cultura Económica.

- Stavrakakis, Y. y Glynos, J. (2008). Encuentros del tipo real. Indagando los límites de la adopción de Lacan por parte de Laclau. En Critchley, S. y Marchart, O. (Comps.), Laclau. Aproximaciones críticas a su obra. Buenos Aires: Fondo de Cultura Económica.

\section{Fuentes documentales}

- Acuña, G. (1997). El marco institucional ambiental para una política de gestión de residuos en países seleccionados de América Latina: análisis a la luz de la experiencia internacional. Santiago de Chile: CEPAL/GTZ.

- Alsogaray, M.J. (1998). Un modelo práctico. Reportaje a María Julia Alsogaray por Mara Bugoni y Carlos Cañás. Aportes para el Estado y la Administración Gubernamental, 12, 9-14.

- Basel Convention. (2013). Parties to the Basel Convention. Recuperado de http://www.basel.int/Countries/Statusofratifications/PartiesSignator ies/tabid/1290/language/en-US/Default.aspx

- Birdshall, N. y Wheeler, D. (1993). Trade Policy and Industrial Pollution in Latin America: Where Are the Pollution Havens? Journal of Environment \& Development, 2, 1. 
- CEPAL. (1995). Medio ambiente y comercio internacional en América Latina y el Caribe. En SELA/UNCTAD, Comercio y medio ambiente. El debate internacional (pp. 63-99). Caracas: Nueva Sociedad.

- Chudnovsky, D. y Chidiak, M. (1996). Apertura, reestructuración productiva y gestión ambiental. Las industrias básicas en la Argentina. Desarrollo Económico, Especial(36), 155-180.

- Chudnovsky, D. y López, A. (1996). Política tecnológica en la Argentina: ¿hay algo más que laissez faire? Redes, 3(6), 33-75.

- Chudnovsky, D. y López, A. (2003). Diffusion of environmentally friendly Technologies by multinational corporations in developing countries. International Journal of Technology Management and Sustainable Development, 2(1), 5-18.

- Gómez-Lobo, A. (1992). Las consecuencias ambientales de la apertura económica en Chile. Colección Estudios CIEPLAN, 35, 85-124.

- HCDN [Honorable Cámara de Diputados de la Nación]. (1991). Diario de Sesiones. $40^{\circ}$ Reunión, continuación de la $11^{a}$ Sesión Ordinaria, Inserción solicitada por el Diputado Blanco, 27 de Setiembre de 1990.

- HSN [Honorable Senado de la Nación]. (1991). Diario de Sesiones. $36^{\circ}$ Reunión, $18^{\circ}$ Sesión Ordinaria, Inserción $2^{\circ}$ solicitada por el Senador Gass. Versión taquigráfica de la reunión del trabajo sobre: Residuos Tóxicos; Dr. Nicolo Gligo Director Unidad Conjunta CEPAL-PNUMA, 30 de Octubre de 1991.

- Kummer, K. (2011). Introducción. En PNUMA y Convención de Basilea, Convenio de Basilea. Protocolo sobre responsabilidad e indemnización por daños resultantes de los movimientos transfronterizos de desechos peligrosos y su eliminación. Textos $y$ anexos. Ginebra: Naciones Unidas.

- Muñoz, H. (1995). Una perspectiva chilena sobre comercio internacional y medio ambiente. En Bec, E., Comercio Internacional y Medio Ambiente. Derecho, Economía y Política (pp. 367-379). Buenos Aires: Espacio-CIEL.

- OECD. (1996). The Global Environmental Goods and Services Industry, París: OECD.

- Ruz, A.M. y Mladinic, H. (2005). Identificación de áreas de oportunidad en el sector ambiental de América Latina y el Caribe. Casos exitosos de colaboración entre industrias para formular alianzas. Serie Medio Ambiente y Desarrollo, 30.CEPAL.

- Schatan, C. (1999). Contaminación industrial en los países latinoamericanos pre y post reformas económicas. Serie Medio Ambiente y Desarrollo, 22. Santiago de Chile: CEPAL, División de Medio Ambiente y Asentamientos Humanos. 
- Scholz, I. (1993). Requerimientos ambientales a productos de consumo en Alemania y sus efectos sobre las exportaciones de los países en desarrollo. Berlín: Instituto Alemán del Desarrollo.

- Sorsa, $P \cdot \frac{36}{\cdot}$ (1995). El medio ambiente en el contexto del comercio internacional. En SELA/UNCTAD, Comercio y medio ambiente. El debate internacional (pp. 101-109). Carcas: Nueva Sociedad.

- Strong, M. (2013): The Founex Conference. Recuperado de http://www.mauricestrong.net/index.php/founex-

conference?showall=1\&limitstart=

- WICEM II. (1991). Second World Conference on Environmental Management: conference report and background papers. Editado por Jan-Olaf Willums y Ulrich Golüke. París: International Chamber of Commerce.

1. Doctor en Ciencia Política. Docente-investigador, Universidad Nacional de Villa María. Córdoba, Argentina. jorgefoatorres@gmail.com

2. Por posmarxismo se hace referencia a aquellos esfuerzos orientados a reformular radicalmente el programa materialista de Karl Marx abrevando en la lingüística estructural y la enseñanza psicoanalítica freudiano-lacaniana. Lo cual no implica la postulación de un antimarxismo sino de una revisión que haga énfasis en el "momento relacional" de la obra marxiana y la crítica al esencialismo filosófico (Laclau y Mouffe, 2000).

3. La utilización de estas denominaciones clasificatorias de los países (desarrollados, subdesarrollados o en desarrollo) no implica la adhesión por nuestra parte a su tenor conceptual sino el seguimiento de los significantes tal y como son presentados en el referente en cuestión. Por tal motivo, cuando se busque hacer énfasis en los aspectos conceptuales vinculados a las asimetrías estructurales en países se utilizarán las denominaciones "países centrales" y "países periféricos" o "países del Norte" y "países del Sur".

4. Los esfuerzos de Maurice Strong, Secretario General de Estocolmo (quien 20 años después sería el Secretario también de la Cumbre de Río de 1992), fueron claves en el logro del compromiso por parte de los PED. Lugar destacado en ese contexto tuvo la "iniciativa Founex", un seminario de expertos de todo el mundo (27 en total entre los que se contaba a Samir Amin) llevado a cabo en la ciudad suiza de Founex en la primavera de 1971 (Strong, 2013).

5. El Convenio de Basilea entró en vigor recién el 5 de mayo de 1992 y, si bien en la actualidad cuenta con al menos 175 países miembro, a marzo de 2013 EE.UU. (el mayor generador de desechos tóxicos del mundo) aún no forma parte del mismo habiendo únicamente firmado el Convenio el 22 de marzo de 1990 
sin haberlo aprobado o aceptado formalmente (Basel Convention, 2013).

6. En los albores de la década los casos testigo elegidos por la unidad conjunta CEPAL/PNUMA fueron la ciudad de Buenos Aires y Cartagena de Indias. Poco tiempo después y en el marco de diversos escándalos por corrupción en los que se vio envuelta la gestión del Intendente Carlos Grosso, los proyectos se mudaron a la ciudad de Córdoba.

7. Como Cartagena de Indias (Colombia), Campinas (Brasil) y Quito (Ecuador) (entre otros: CEPAL, 1995; Acuña, 1997). En tal contexto, en el año 1996 la Municipalidad de Córdoba sancionó su Ordenanza $n^{\circ} 9612$ sobre Residuos bajo la intendencia de Rubén Martí y la gestión en la Subsecretaría de Medio Ambiente del Ing. Alberto Ferral.

8. Respecto de las categorías intermedias como noción clave para la propuesta epistemológica del análisis político del discurso ver entre otros: Saur (2008).

9. En otras palabras, no se piensa en los procesos de significación como instancias secundarias o efectos de relaciones sociales originadas en tales o cuales planos materiales, ya sea que esa materialidad sea endilgada a ciertas relaciones de carácter económico, jurídico o cultural. Por el contrario, los procesos de significación son entendidos desde nuestra perspectiva como elementos que estructuran la realidad.

10. "Para [la lógica de la sobredeterminación] el sentido de toda identidad está sobredeterminado en la medida en que toda literalidad aparece constitutivamente subvertida y desbordada; es decir, en la medida en que, lejos de darse una totalización esencialista o una separación no menos esencialista entre objetos, hay una presencia de unos objetos en otros que impide fijar su identidad" (Laclau y Mouffe, 2004, p. 142).

11. Cabe advertir que este proceso no es lineal sino que cada una de estas instancias informa y es informada a su vez por las demás a lo largo del trabajo.

12. Excepto aquellas consideradas indispensables (en el cuerpo del texto) o altamente ilustrativas (en notas al pie).

13. La OCDE la ha identificado, en términos generales, como aquella destinada a suministrar equipamientos y servicios destinados al control de la contaminación, la reducción y el manejo de residuos (OECD, 1996).

14. Al respecto ver: Foa Torres (2014).

15. Como afirmaba Michel Foucault la noción de tecnologías, refiere al abordaje de diferentes modos de conocer que en tanto "juegos de verdad específicos, relacionados con técnicas específicas [...] los hombres utilizan para entenderse a sí mismos". Cada tecnología representa "una matriz de la razón práctica" y, entre 
ellas, se cuenta a las tecnologías de poder como aquellas que "determinan la conducta de los individuos, los someten a cierto tipo de fines o dominación, y consisten en una objetivación del sujeto" (Foucault, 2008, p. 48). Si bien en líneas generales adherimos a tales conceptos, desde nuestra visión las tecnologías gubernamentales de poder se inscriben en lógicas políticoideológicas capaces de sobredeterminar un sinnúmero de prácticas sociodiscursivas. En tal sentido, las tecnologías gubernamentales permiten abordar al Estado como "el correlato de una manera determinada de gobernar" y no como un simple monstruo o gendarme puramente represor (Foucault, 2012, p. 21).

16. Como advertía Imme Scholz en Requerimientos ambientales a productos de consumo en Alemania y sus efectos sobre las exportaciones de los países en desarrollo, publicado en el año 1993 por el Instituto Alemán de Desarrollo: "Los requerimientos al producto vienen cobrando mayor importancia en Alemania y en la Comunidad Europea. Dado que esos requerimientos se aplican tanto a las mercancías del país como a las importadas, pueden convertirse también en barreras comerciales para-arancelarias y obligar a los socios comerciales a adaptarse a ellos [...] Los requerimientos al producto pueden tener también el objetivo de influir de manera indirecta sobre los procedimientos de fabricación o explotación en el extranjero que escapan al campo de acción del parlamento o de la institución normativa nacional. En este caso tienen el mismo efecto que barreras comerciales paraarancelarias. Este instrumento se aplica particularmente en el caso de los problemas ambientales globales o regionales" (Scholz, 1993, I).

17. Como también señalaba Imme Scholz en el informe citado: "Vistas en su totalidad, las condiciones de acceso que tendrán en el futuro los exportadores de terceros países al mercado europeo resultan poco transparentes. En vista de la situación, se aconseja a las empresas de países en desarrollo interesadas en ocupar a largo plazo segmentos del mercado europeo que incorporen estándares ambientales en sus estrategias de producción y que se orientan a largo plazo por un nivel exigente de protección ambiental, empezando a nivel de requerimientos al producto en las ramas dirigidas a la exportación" (1993, iv y p. 22).

18. "Trade Policy and Industrial Pollution in Latin America: Where Are the Pollution Havens?".

19. Por citar solo algunos: Gómez-Lobo (1992), CEPAL (1995), Muñoz (1995), Chudnovsky y Chidiak (1996), Schatan (1999).

20. "La evidencia anecdótica de Chile sugiere varias razones por la cuales la eliminación de las barreras a la importación de nuevas tecnologías y al

capital extranjero puede conducir a la importación de las normas de contaminación de los países industrializados. Una vez que estos estándares más altos se introducen (a pesar del hecho de que pueden ser demasiado altos desde el punto de vista de la demanda local de calidad ambiental), las grandes empresas multinacionales tienden a presionar por su aplicación con el fin de 
reducir la ventaja de costos de las firmas locales de menor tamaño" (Birdsall y Wheeler, 1993, p. 147).

21. Como señalaba Maurice Strong en su discurso ante la Segunda Conferencia Mundial Industrial sobre Gestión Ambiental (WICEM II, por sus siglas en inglés: World International Conference on Environmental Management) de 1991: "La Experiencia de Europa Occidental y Japón ha demostrado que la mejora del medio ambiente puede ser totalmente compatible con altas tasas de rendimiento económico y de hecho puede hacer una contribución positiva a ese rendimiento. En el otro extremo, la experiencia de la Unión Soviética y otros países de Europa Central y del Este es que algunos de los peores ejemplos de negligencia ambiental y el deterioro han sido los subproductos de la gestión económica y de rentabilidad igualmente desastrosa" (1991, p. 52).

22. En este sentido Heraldo Muñoz en el documento "Una perspectiva chilena sobre comercio internacional y medio ambiente" señalaba que: "la evidencia del caso chileno no apoya la tesis de que el libre comercio transforma a países que impulsan procesos de apertura económica en refugios para la contaminación y degradación de los recursos naturales. En el sector industrial -según una fuente- la apertura chilena al comercio exterior ha tenido un impacto positivo sobre el medio ambiente porque industrias contaminantes se han expandido a tasas inferiores a las que contaminan menos" (1995, p. 377).

23. Como señalaba la CEPAL en un documento institucional del año 1995: "[...] las exigencias provenientes del exterior, evidentemente llegan de modo inmediato a las firmas que exportan -directa y, eventualmente, según el tipo de regulación, indirectamente- o que se financian en el exterior -solo las de mayor tamaño y/o filiales de ET [Empresas Transnacionales]-. En el caso de las ET, la presión de las casas matrices -que, en muchos casos, pueden verse involucradas en dificultades legales en sus países de origen si generan problemas de contaminación en los países donde se radican- lleva a que crecientemente se adopten prácticas comunes en todas las filiales; estas prácticas, muchas veces, también implican mayores presiones para los proveedores locales de dichas filiales" (1995, p. 50).

24. El aprovechar de manera ilimitada las ventajas de costos comparativos más bajos debido a estándares ambientales menos restrictivos o no existentes sería contraproductivo, ya que cimentaría la brecha tecnológica entre los países industrializados y los países en desarrollo (Scholz, 1993, III).

25. Hacemos referencia al dumping ecológico en el sentido en que lo conceptualizó la cooperación alemana en tanto aprovechamiento de los menores costos de la producción por parte de los países cuyos estándares ambientales sean inferiores a los de los países importadores.

26. En un sentido similar la por entonces Secretaria de Recursos Naturales y Desarrollo Sustentable de la Nación, la ingeniera María Julia Alsogaray, precisaba que: "Lo ambiental no es un tema para imponer por ley marcial, a tambor batiente. Es un tema 
para normas que se van a ir decantando. Porque lo que hay son experiencias. Eso es lo que nosotros tratamos: condensar experiencias del exterior y volcarlas en el país" (Alsogaray, 1998, p. 13, énfasis agregado).

27. Como la certificación de normas ISO (Organización Internacional para la Estandarización, en inglés: International Organization for Standardization) de estandarización industrial. Normas que poseen, en términos de Jenifer Clapp, un carácter transnacional, técnico y de facto en tanto son capaces de burlar las instancias democrático-estatales para la formulación de estándares de producción susceptibles de hacerse obligatorios en cualquier rincón del mundo (Clapp, 1998).

28. "Hubo un avance notable en este sentido. Por eso nosotros hacemos fuertes campañas para que las empresas traten de certificar según normas internacionales, ya que tal certificación, al ser independiente del poder político, no está sujeta a eventuales presiones" (Alsogaray, 1998, p. 9).

29. En este caso del abogado Daniel Silva por entonces consultor de CEPAL, que señalaba que: "Además, no es fácil imaginar un sistema integral para la Argentina en el término de los próximos años. Esto de alguna manera tiene que ver lo que estimo es un vicio del pensamiento de los planificadores en nuestro país y en la región, en el sentido de que es el Estado el que debe brindar necesariamente las soluciones a los problemas que le plantean los agentes económicos privados. $Y$ en este sentido la propuesta del equipo de consultores plantea una originalidad: quien debe dar la respuesta no es el Estado sino los agentes contaminantes. El Estado lo que debe hacer es fiscalizar, identificar líneas de crédito, implementar un sistema de promociones de asistencia, orientar la planificación estatal, ayudar y orientar la planificación privada. Pero el principio básico es que el manejo de residuos tóxicos y peligrosos debe ser soportado por quienes los generan y son, en definitiva, los que se benefician con dicha generación" (HSN, 1991, p. 3547, énfasis agregado).

30. El mismo think tank CENIT (Centro de Investigaciones para la Transformación) daba cuenta de esta situación, en un artículo específicamente referido al tema titulado "Política tecnológica en la Argentina: ¿hay algo más que laissez faire?" de Chudnovsky y López del año 1996 que advertía que: "[...] los gobiernos argentinos hace tiempo han optado por desentenderse del tema de СуT, dejando a la mayoría de las instituciones del complejo libradas a su propia suerte y confiando el proceso de innovación tecnológica a las señales del mercado y las decisiones privadas. Aunque practicado durante casi toda la historia del país [...], el laissez faire en materia tecnológica de la actual administración encontraría sus fundamentos en la teoría económica ortodoxa que, con escasas excepciones, considera a la tecnología como una variable exógena- $y$, sobre todo, en las recomendaciones de política del llamado Consenso de Washington, que priorizan la liberalización comercial y la promoción de la inversión extranjera directa (IED) como instrumentos básicos para lograr la modernización tecnológica" (1996, pp. 33-34). 
31. Es por eso que se ha preferido hablar de lo "transnacional" antes que de lo "multinacional". Mientras este último término da cuenta sencillamente de una pluralidad de naciones, el primero hace referencia al atravesamiento de éstas, a aquello que desborda las fronteras y tiende a borrarlas. Para la vocación transnacional no hay límites estatales insuperables, no existen ámbitos soberanos intocables sino oportunidades para la expansión.

32. Como señala Mónica Peralta Ramos el resultado de este proceso fue un: "[...] crecimiento inigualable de la concentración y centralización de capitales a nivel mundial y una reversión de la tendencia descendente de la tasa de ganancia del capitalismo estadounidense desde finales de la década de 1980" (2007, p. 43).

33. El principio fue pensado en sus orígenes para ser aplicado a nivel de cada Estado nacional, constituyéndose en un mecanismo de transferencia de costos ambientales hacia el causante de los daños ambientales, con el objetivo económico de: "[...] internalizar los costos externos de la protección ambiental. Al mejorar [...] la función informativa del sistema de precios ('precios que dicen la verdad ecológica') se quiere lograr que el consumidor pueda escoger su opción de compra según criterios ambientales" (Scholz, 1993, p. 2). Pero con la globalización de las políticas de protección ambiental, todo portador de riesgo ambiental o causante de daño ambiental, provenga del lugar que provenga y de la industria que sea, deberá ser tratado por igual a la hora de transferirse los costos ambientales de su actividad productiva.

34. Justamente el sintagma "gestión ambientalmente adecuada".

35. Cabe señalar que, en el marco del método retroductivo, la hipótesis es el lugar de anudamiento entre referente empírico y fundamentos ontológicos. Por tanto lo hipotetizado resultante del trabajo de indagación, adviene como inferencia solo al momento en que su contenido está ya presente en el edificio conceptual que permitió comprender el problema.

36. La autora, en aquel entonces se desempeñaba como Economista Superior en la División de Comercio Internacional del Banco Mundial. De nacionalidad finlandesa fue responsable por el Banco Mundial en la Ronda de Uruguay en temas de comercio y medio ambiente.

Para citar este artículo: Foa Torres, J.G. (2016). Lógica de la gestión ambientalmente adecuada y patrón de desarrollo sustentable en América Latina: el tema emblemático de las políticas de residuos peligrosos en Argentina en los años 90. Revista Luna Azul, 42, 293-318. Recuperado de http://200.21.104.25/lunazul/index.php?option=com_content\&view=artic le\&id $=140$ 